\title{
Histological Scoring for Nonalcoholic Fatty Liver Disease - A Study in 55 Cases
}

\author{
REHANA KHANAM, ${ }^{1}$ MUHAMMAD KAMAL, ${ }^{2}$ FAUZIA JAHAN, ${ }^{1}$ P. K. GHOSH ${ }^{3}$
}

\begin{abstract}
:
Background: Steatohepatitis is a pattern of liver injury that may be seen in alcoholic or nonalcoholic liver disease. This morphological changes may occur with obesity, diabetes, the use of certain drugs or it may be idiopathic. The main histopathological features of nonalcoholic steatohepatitis (NASH) include hepatocellular steatosis and ballooning, mixed acute and chronic lobular inflammation and fibrosis. The recently developed histological scoring system for NAFLD by the NASH Clinical Research Network (NASH CRN) is becoming increasingly popular. The purpose of this study was to develop grading and staging system and was based on liver biopsies from 50 patients with Nonalcoholic Steatohepatitis from BSMMU and other clinics of Dhaka city.

Objective: This study was done to analyze the histological spectrum and to develop grading and staging system in patients with nonalcoholic fatty liver disease.

Methods: This cross sectional study was carried out at Bangabandhu Sheikh Mujib Medical University (BSMMU), Dhaka, Bangladesh. A total of 55 patients with non-alcoholic fatty liver disease (NAFLD) who fulfilled the inclusion criteria were selected for the study. Liver biopsy was done at hepatology department of BSMMU, and different clinics of the Dhaka City from july 2006 to june 2007. The data of patients along with histopathological reports were studied.

Results: Out of total 55 cases of Nonalcoholic Fatty Liver Disease 33 were male (63.63\%) and 22 were female (40\%). Mean age of the patient was $37.81 \pm 10.80$ years. 35 (63.63\%) patients were obese. 2 patients (3.63\%) were diabetic. Among 55 patients NAFL (steatosis) were diagnosed in 6 patients (10.90\%) and NASH in 49 patients (89.09\%). Forty two (16.36\%) of the 46 Definite NASH biopsy specimen exhibited fibrosis and three had cirrhosis.
\end{abstract}

Key words: Nonalcoholic fatty liver, Steatohepatitis, Cirrhosis.

\section{Introduction:}

Nonalcoholic fatty liver disease (NAFL) is a morphological pattern of liver injury due to fat accumulation within hepatocytes in the absence of significant alcohol use or other known liver diseas. NAFLD encompasses a wide spectrum of liver diseases ranging from simple fatty liver (steatosis) to non-alcoholic steatohepatitis (NASH ) and eventually cirrhosis (irreversible advanced scarring of the liver ). ${ }^{1}$

The grading and staging approach have been applied to asses the severity of fatty liver disease.

According to The Pathology Committee of the NASH Clinical Research Network a NAFLD activity score (NAS) system that addresses the full spectrum of lesions of NAFLD and proposed

1. Asst. Professor, Dept. of Pathology, Bangladesh Medical College, Dhaka

2. Professor \& Chairman, Dept of Pathology, BSMMU, Dhaka

3. Professor \& Head, Dept of Pathology, Bangladesh Medical College, Dhaka

Correspondence: Dr. Rehana Khanam, Asst. Professor, Dept. of Pathology, Bangladesh Medical College, Dhaka a NAFLD activity score (NAS) for use in clinical trials. Four features were evaluated semiquantitatively: steatosis ( 0-3), Lobular inflammation, (0-2), hepatocellular ballooning (0-2), and fibrosis (0-4). The individual scores then added to produce an overall "NAFLD Activity Score" (NAS). ${ }^{2}$ There is also recognition of portal fibrosis as a separate pathway for disease progression. $^{2}$

With this view the present study was carried out to determine scoring which includes probable or definite NASH (score $>5$ ), Uncertain (score 3-4) and Not NASH (<2). The cases were collected from different institutes and pathologic laboratories of Dhaka city.

\section{Material and Method:}

This is a cross sectional study carried out at the Department of Pathology, Bangabandhu Sheikh Mujib Medical University (BSMMU), Dhaka from July 2006 to June 2007.

A total of 55 patients were selected for the study. These patients were clinically, biochemically and sonologically diagnosed as suffering from non-alcoholic fatty liver disease. 
Liver biopsy was done at hepatology department of BSMMU and different clinics of the Dhaka City.

Histopathological evaluation of liver biopsy was done to see the Lobular architecture, Hepatic cell plate, Steatosis, Lobular inflammation, Hepatocyte ballooning and fibrosis.

\section{Grading:}

Individual scores of steatosis, lobular inflammation and hepatocyte ballooning added to produce an overall 'NAFLD Activity Score: (NAS). NAFLD activity score $=>5$ then the histological diagnosis will be Probable or definite NASH; 34 - Uncertain and $<2$ - Not NASH.

\section{Staging:}

Staging was used to evaluate the intensity of necroinflammatory activity and extent of fibrosis. The staging score Stage 1 ( perisinusoidal or periportal fibrosis) which is further divided into 1A, Mild, zone 3, perisinusoidal ; 1B Moderate, zone 3, perisinusoidal ; 1C Portal/ periportal fibrosis only; Stage 2, Perisinusoidal and portal/periportal fibrosis; Stage 3, Bridging fibrosis; Stage 4 Cirrhosis.

\section{Result:}

A total of 55 cases were included of whom 33 were male $(60 \%)$ and 22 were female (40\%). Among 55 patients NAFL (steatosis) were diagnosed in 6 patients $(10.90 \%)$ and NASH in 49 patients $(89.09 \%)$.

\section{Histological evaluation of liver biopsies}

Liver biopsy was done in all fatty liver patients. Changes in liver according to Kleiner et al 2005 classification is shown in table 4.6.

Table-I

Histological parameter of $\mathrm{NASH}$

\begin{tabular}{|c|c|c|c|c|c|}
\hline \multicolumn{6}{|c|}{ Histological } \\
\hline \multirow{2}{*}{\multicolumn{2}{|c|}{ Diagnosis }} & \multicolumn{4}{|c|}{ Histological Parameter } \\
\hline & & \multicolumn{4}{|c|}{$\begin{array}{c}\text { Steatosis Lobular Hepatocyte Fibrosis } \\
\text { inflammation ballooning }\end{array}$} \\
\hline \multicolumn{2}{|c|}{ NAFL $(\mathrm{N}=6)$} & $100 \%$ & 0 & 0 & 0 \\
\hline \multirow[t]{3}{*}{ NASH } & $\begin{array}{l}\text { Definite NASH } \\
(\mathrm{N}=46)\end{array}$ & $\begin{array}{l}100 \% \\
(\mathrm{~N}=46)\end{array}$ & $\begin{array}{l}97.82 \% \\
(\mathrm{~N}=45)\end{array}$ & $\begin{array}{c}100 \% \\
(\mathrm{~N}=46)\end{array}$ & $\begin{array}{l}76.36 \% \\
(\mathrm{~N}=42)\end{array}$ \\
\hline & $\begin{array}{l}\text { Uncertain NASH } \\
(\mathrm{N}=4)\end{array}$ & $\begin{array}{l}\mathrm{H} 50 \% \\
(\mathrm{~N}=2)\end{array}$ & $\begin{array}{l}75 \% \\
(\mathrm{~N}=3)\end{array}$ & $\begin{array}{l}50 \% \\
(\mathrm{~N}=2)\end{array}$ & $\begin{array}{c}75 \% \\
(\mathrm{~N}=3)\end{array}$ \\
\hline & $\begin{array}{l}\text { No NASH } \\
(\mathrm{N}=5)\end{array}$ & $\begin{array}{l}100 \% \\
(\mathrm{~N}=5)\end{array}$ & 0 & 0 & 0 \\
\hline
\end{tabular}

Amongst the features which have been assessed in fatty liver disease in the present study agreement is generally good for steatosis, moderate for ballooning and less good for lobular inflammation and Mallory's hyaline.
Table-II

Histological scoring for NAFLD

\begin{tabular}{lcc}
\hline NAS-score Component & \multicolumn{2}{c}{ NAS score } \\
\cline { 2 - 3 } & Number & Percentage (\%) \\
\hline Steatosis & 02 & $3.64 \%$ \\
$0=<5 \%$ & 9 & $16 . .36 \%$ \\
$1=5 \%-33 \%$ & 16 & $29.09 \%$ \\
$2=33 \%-66 \%$ & 28 & $50.90 \%$ \\
$3=>66 \%$ & & \\
Lobular Inflammation & 06 & $10.90 \%$ \\
$0=$ none & 15 & $27.27 \%$ \\
$1=<2$ foci per x 200 field & 32 & $58.18 \%$ \\
$2=2-4$ foci per x 200 field & 2 & $4.34 \%$ \\
$3=>4$ foci per x 200 field & & \\
Ballooning & 06 & $10.9 \%$ \\
$0=$ none & 0 & 0 \\
$1=$ Few ballooned cells & 49 & $89.09 \%$ \\
$2=$ many cells/Prominent & & \\
balloning & & \\
\hline
\end{tabular}

\section{Steatosis}

There were 55 cases. $6(10.90 \%)$ cases with steatosis without inflammation and ballooning degeneration. Scoring was done more precisely for steatosis. Among them score 1 was $16.36 \%$ (n-9), score 2 was $29.09 \%$ (n-16) and score 3 was $50.90 \%$ (n28).

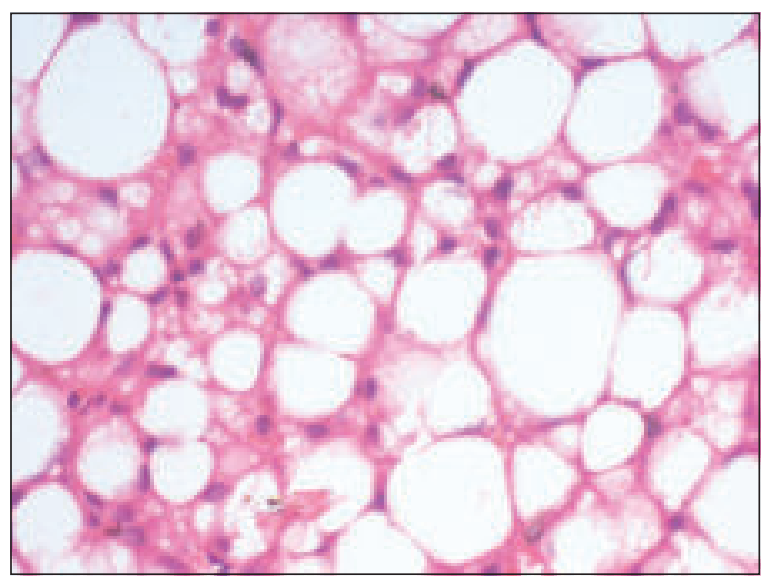

Fig.-1: Photomicrograph show macrovesicular steatosis (Score-3, case No. 45, H\&E stain $x 40$

Lobular Inflammation

In NAFLD group, score 1 was seen in $27.27 \%$ (n-15), score 2 in $58.18 \%(\mathrm{n}-32)$ and score 3 was in $4.34 \%(\mathrm{n}-2)$ cases.

\section{Hepatocyte ballooning}

In NAFLD group 6 cases were found having score 0 . Score 2 was seen in $89.09 \%(n=49)$ patients.

\section{Fibrosis}

Fibrosis was evaluated through $\mathrm{H} \& \mathrm{E}$ and Masson's trichrome stains. Details of fibrosis is shown in Table-4.8. Forty two 


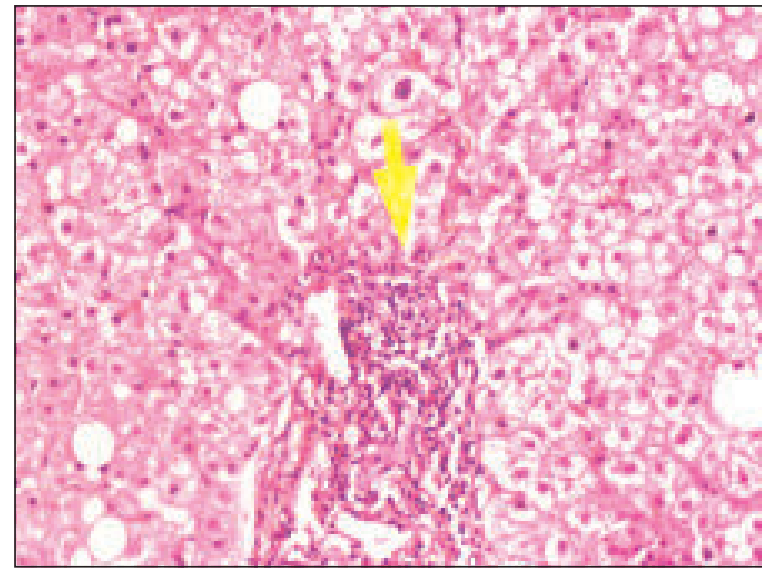

Fig.-2: Lobular Inflammation

(16.36\%) of the 46 Definite NASH biopsy specimen exhibited fibrosis. Stage 1A was seen in 8.69\%\% (n-4) in Masson's trichrome stain, stage $1 \mathrm{~B}$ was seen in $2.17 \%(\mathrm{n}-1)$, stage $1 \mathrm{C}$ was seen in $10.86 \%$ (n-5) and stage 2 was seen in M.T stain but in $\mathrm{H}$ $\&$ E stain these cases were evaluated as stage 0 or 1C. Stage 3 was observed in $13.04 \%(n-6)$ cases of which 4 biopsy specimen

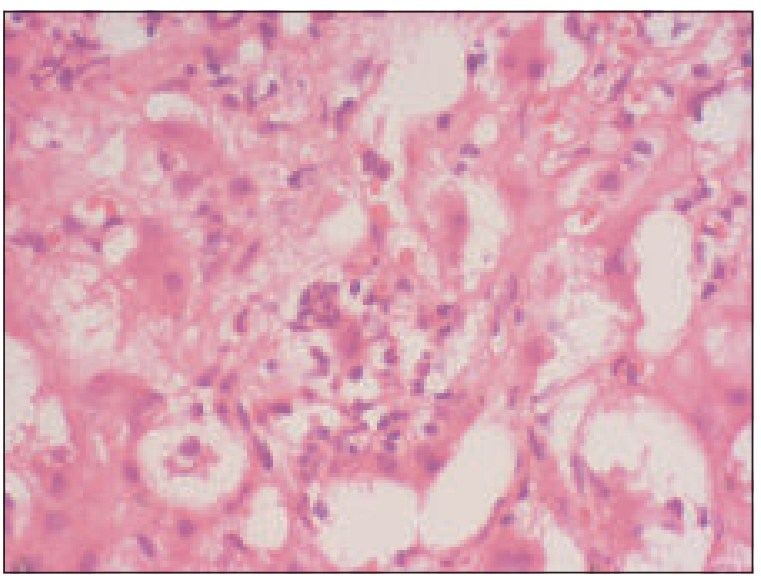

Fig.-3: Hepatocyte Ballooning

ware diagnosed as stage 3 in $\mathrm{H} \&$ E. Stage 4 ( cirrhosis) was seen in $6.52 \%$ ( $n-3$ ) in Masson's trichrome stain. Fibrosis score for stage 1 were extended to include a distinction between delicate (1A) and dense (1B) perisinusoidal fibrosis and to detect portal-only fibrosis, without perisinusoidal fibrosis ( stage $1 C)$.

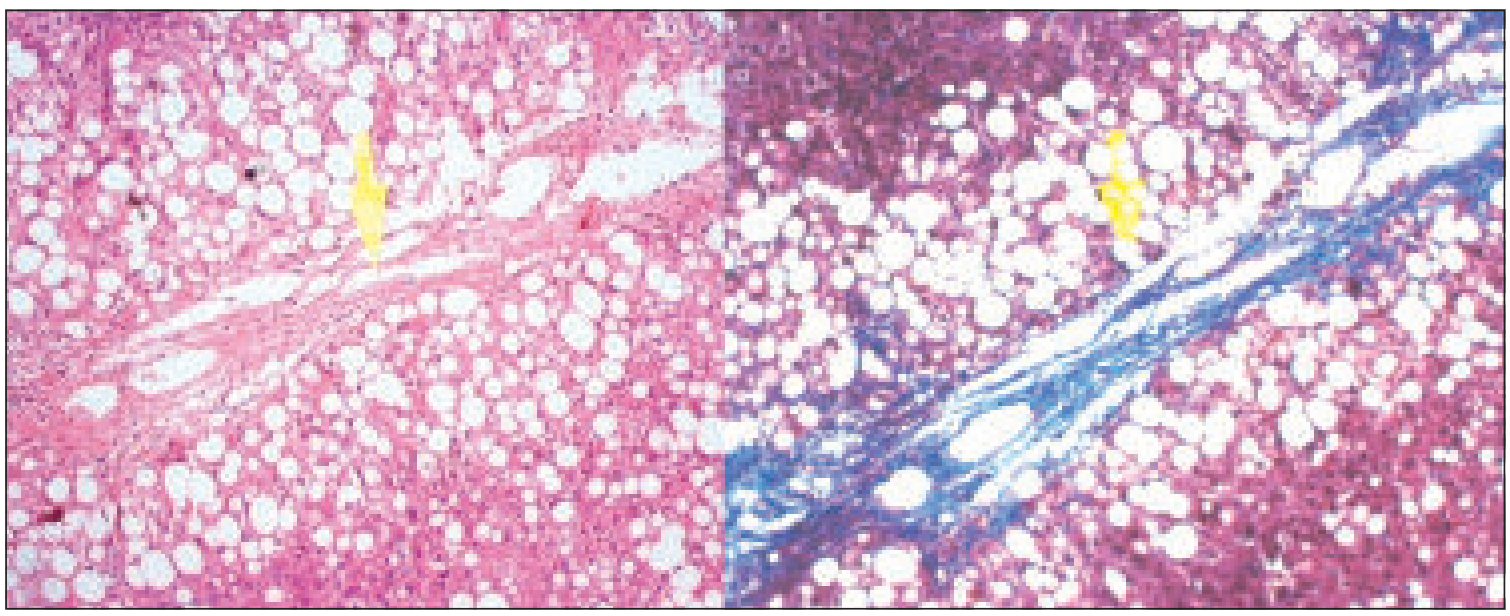

Fig.-4: Bridging Fibrosis

Table-III

Fibrosis Stage

Fibrosis Component

\begin{tabular}{lc}
\multicolumn{2}{c}{ Stage } \\
\hline As scored in & As scored in \\
H \& E stain & Masson's Trichrome stain
\end{tabular}

Perisinusoidal or periportal

1A Mild,zone 3,perisinusoidal

1B Moderate, Zone 3,perisinusoidal

1C Portal/periportal fibrosis only

2. Perisinusoidal and portal/periportal Fibrosis

Masson's Trichrome stain

3. Bridging fibrosis

4. Cirrhosis

$\begin{array}{cc}0 & 4(8.69 \%) \\ 0 & 1(2.17 \%) \\ 23(50 \%) & 5(10.86 \%) \\ 1(2.17 \%) & 26(56.52 \%) \\ 4(8.69 \%) & 6(13.04 \%) \\ 2(4.34 \%) & 3(6.52 \%)\end{array}$




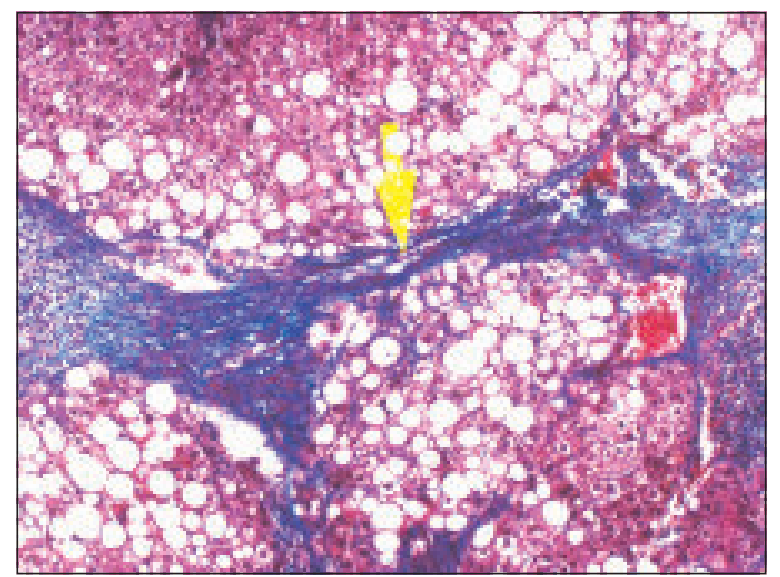

Fig.-5: Photomicrograph shows cirrhosis (case No. Masson's Trichrome stain x 10)

\section{Discussion:}

There has been great interest and anxiety regarding nonalcoholic fatty liver disease (NAFLD). It is a growing problem in the Western hemisphere. In USA the prevalence among general population is $20 \% .^{3}$ Awareness about NAFLD is increasing in the Asia-Pacific region ${ }^{4}$ A prevalence of NAFLD in this area has been estimated ranging from $20-30 \%$ (4). NAFLD represents a spectrum of clinicopathologic syndromes and represents with varying risk of cirrhosis. ${ }^{5}$ Bangladesh is not excluded from this crisis. The aim of the present study was to evaluate the histomorphological features of NAFLD with grading and staging of the disease. A total of 55 NAFLD cases were selected on the basis of clinical and biochemical feature of the liver disease. Among 55 patients, NAFL (simple steatosis) was diagnosed in 6 $(10.9 \%)$ and NASH in $49(89.09 \%)$ cases. In developing countries like Bangladesh the incidental finding of NAFLD is increasing because of change in life style, food habit, and lack of conciousness. In Bangladesh many children and adolescents with suspected liver disease are seen by hepatologists. A significant number of cases of "cryptogenic cirrhosis" are found which may represent end stage of NAFLD.

During the 12 month of the study, the subject's liver biopsy specimens were submitted to the laboratory as "routine" cases were not specified as "study biopsies" to avoid possible biased interpretation. One hematoxylin and eosin stained slide and one Masson's trichrome stained slide were studied. Different studies when reporting the histologic findings in patients with NASH, only steatosis was universally present as an diagnostic features . In the present study out of 55 liver biopsy specimen all i.e. 100\% biopsies had steatosis. Steatosis as part of NAFLD is usually macrovesicular in type, perivenular in distribution. Steatosis are scored according to NAS -score, modified by Kleiner et $a l$ in 2005 . In the present study $2(3.64 \%)$ biopsies reveal steatosis score 0 ( $<5 \%$ hepatocytes), score 1 (5\%- 33\% hepatocytes) in $9(16.36 . \%)$ biopsies, score 2 (33-66\% hepatocytes) in $16(29.09 \%)$ biopsies and score 3 ( $>66 \%$ hepatocytes) in $20(50.90 \%)$ biopsies.

In the present study at BSMMU lobular inflammation was present in all but $6(10.90 \%)$ patients. $15(27.27 \%)$ biopsies had score 1 , 32 (58.18\%) had score 2 and 2 (4.34\%) had score 3. In a similar study it was observed that lobular inflammation was identified in most patients and score 2 was present in more than half biopsies. ${ }^{6}$

Regarding ballooning score 1 was not found in this study. Score 2 was seen

in $49(89.09 \%)$ patients. Ballooning was not seen in 6(10.90\%) patients .In a similar study ballooning was seen in 100 biopsies ${ }^{7} \mathrm{~A}$ hallmark of NASH is the development of a specific form of fibrosis where a collagenous matrix is deposited along the hepatocytes in the space of Disse. In the most prominent cases, individual hepatocytes appear to be outlined by a rim of collagen, giving the liver a chicken wire appearance.

In present study 42 of the 46 definite NASH patients exhibited fibrosis . When using Masson's Trichrome stained slides, scored stage $1 \mathrm{~A}$ was $8.69 \%(\mathrm{n}-4)$, stage $1 \mathrm{~B}$ was $1.8 \%$ $(n=1)$, stage $1 \mathrm{C}$ was $10.86 \%(n=5)$ and stage 2 was seen in $56.52 \%$ (n-26). But in H \& E stain these score were evaluated as stage 0 or $1 \mathrm{C}$. Stage 3 was $13.04 \%$ (n-6) in M.T stain and in $\mathrm{H} \& \mathrm{E}$ stain they were evaluated as either $0,1 \mathrm{C}$ or 2 . Stage 4 was $6.52 \%(n=3)$. In H\&E staining stage 4 was $4.34 \%$ $(n=2)$. But cirrhosis was $6.52 \%(n=3)$. In the study by Brunt et al 2004. He studied 30 patients. 19 of the 30 biopsy specimens exhibited fibrosis $(63 \%)$, scored as stage 1 in 7 $(23 \%)$, stage 2 in $4(13 \%)$ and stage 3 in $8(27 \%)$.

\section{Conclusion:}

In our country, NAFLD have biochemical and clinical features similar to those observed in other countries. We have designed and validated a semiquantitative scoring system that is useful for assessing the range of histological features of NAFLD. The system is simple and require only routine histochemical stains ( $\mathrm{H} \& \mathrm{E}$ and Masson trichrome stain).

Grading and staging was determined by analyzing the histological features that can help for prognostic and treatment purpose.

From the present study it was concluded that Masson's trichrome stain is required for detection of collagen deposition in NAFLD cases. The staging will be increased 
in Masson's trichrome stain in comparison to staging in H\&E stain.

\section{Conflict of Interest : None}

\section{References:}

1. LudwigJ, Viggiano TR, McGill DB, Oh BJ. Nonalcoholic steatohepatitis: Mayo Clinic experiences with a hitherto unnamed disease. Mayo Clinic. Proc, 1980; 55: 434-438.

2. Kleiner DE, Brunt EM, VAN Natta $M$ et al. Design and validation of a histological scoring system for nonalcoholic fatty liver disease. Hepatology 2005; 41: 1313-1321.

3. Hubscher SG. Histological assessment of nonalcoholic fatty liver disease. Histopathology, 2006; 49: 450-465.
4. Omagri k. Kadokawa Y, Masuda J et al. fatty liver in nonalcoholic, non-overweight Japanese adults: incidence and clinical characteristics. J Gasteroenterol Hepatol, 2002; 17: 1098-105.

5. Sanyal AJ, Campbell-Sargent C, Mirshahi F et al. Non alcoholic steatohepatitis. Association of insulin resistance and mitochondrial abnormalities. Gastroenterology 2001; 120: 1183-1192.

6. Crawford JM. Liver and Biliary Tract in Robins and Cotran pathologic Basis of Diseases. $7^{\text {th }}$ edition, kumar v, abbas AK, and Fausto N (eds). Saunders Company, Philadelphia. 2004. Pp. 878.

7. Brunt E M.Non-alcoholic Steatohepatitis: Definition and Pathology. Seminar in Liver Disease, 2001; 21:3-16. 